\title{
Sprache im Dienste der Aggression: Verbales und nonverbales aggressives Verhalten in politischen Fernsehdiskussionen am Beispiel von Maybrit IIIner'
} Celem pracy jest zbadanie werbalnego i niewerbalnego agresywnego zachowania na
przykładzie telewizyjnych dyskusji politycznych. Analizie poddano fragmenty pro-
gramu Maybrit Illner. Uwzględnione zostały przy tym prozodyczne cechy wypowie-
dzi oraz mimika, gestykulacja i mowa ciała mówcy i słuchacza, a na płaszczyźnie
językowej - strategie konwersacyjne, typ illokucyjny, formy adresatywne oraz szcze-
gólne środki morfologiczne, składniowe i semantyczne. Część analityczną poprzedza
krótkie wprowadzenie terminologiczne.

Der Aufsatz setzt sich zum Ziel, verbales und nonverbales aggressives Verhalten am Beispiel politischer Fernsehdiskussionen zu untersuchen. Einer genauen Analyse werden Ausschnitte aus der Sendung Maybrit Illner unterzogen. Berücksichtigt werden dabei prosodische Eigenschaften der Äußerungen sowie Mimik, Gestik und Körperhaltung des Sprechers bzw. des Hörers und auf der verbalen Ebene - Konversationsstrategien, Illokutionstyp und Anredeverhalten sowie morphologische, syntaktische und semantische Besonderheiten. Dem analytischen Teil geht eine kurze terminologische Einführung voraus.

The aim of this paper is to investigate verbal and non-verbal aggressive behaviour on the basis of political TV debates such as Maybrit Illner. The analysis includes prosodic aspects of a statement, facial expressions, gestures and body language of both the speaker and hearer as well as conversational strategies, illocution, forms of ad-

1 Der vorliegende Aufsatz stützt sich auf Ergebnisse meiner Untersuchungen im Forschungsprojekt „Sprache als Waffe. Grammatik der Unhöflichkeit“, das im Rahmen des Collegium PONTES 2007 von Prof. Dr. Beata Mikołajczyk betreut wurde. 
Agnieszka Poźlewicz

dress and characteristic morphological, syntactic and semantic means. The analytical part is preceded by a short terminological introduction.

Der vorliegende Aufsatz setzt sich zum Ziel, Strategien sprachlicher Aggression am Beispiel politischer Fernsehdiskussionen zu ermitteln. Da aggressives Verhalten sich nicht allein verbal manifestiert, werden bei der Analyse auch Aspekte der nonverbalen Kommunikation berücksichtigt. Die empirische Basis der durchgeführten Untersuchung bilden sechs Folgen der politischen Talksendung Maybrit Illner, die von April bis Juli 2007 vom ZDF ausgestrahlt wurden. Die Moderatorin, deren Namen das Programm trägt, lädt Prominente aus Politik, Wirtschaft und Gesellschaft ein, die im Studio live über brisante Themen diskutieren. Im Folgenden wird in einer kurzen Einführung auf die wichtigsten Begriffe eingegangen. Den Hauptteil des Aufsatzes bildet die Analyse einer Reihe von Sendungsausschnitten, anhand derer ausgewählte Mittel verbaler und nonverbaler Aggression exemplifiziert werden.

Dazu wird zunächst von der terminologischen Eingrenzung der Bezeichnung ,politische Fernsehdiskussion“ ausgegangen. In Anlehnung an die Diskurstypen-Taxonomie von David Holdcroft (1979) ist zunächst der Terminus ,Diskussion“ als Meinungsaustausch mehrerer Personen zu definieren, die ihre Rollen von Sprecher und Adressaten wechseln, wobei ihnen bei entgegengesetzten Interessen eine gleiche Verteilung des Rederechts zusteht (HUNDSNURSCHER 1994:214f.). Eine politische Diskussion dient nach HOFFMANN (1982:51) der „Informationsvermittlung, Meinungsdarstellung sowie kritischer Problematisierung politischer Handlungen und Auffassungen“. Daher wird die Argumentationshandlung zum „Kern der Politikerbeiträge“ (HoFFMANN 1982:49). Hinzuzufügen bleibt, dass man den Begriff ,Politiker" weit fassen sollte, d.h. als Personen, die ,in politische Handlungen oder Ereignisse verwickelt, durch eine Partei, Gruppe oder Institution, d.h. eine Form von Interessenvertretung legitimiert, eine Funktion ausüben, die über die bloße Betroffenheit durch politische Maßnahmen hinausgeht" (HoFFMANN 1982:16).

Spezifisch für eine Fernsehdiskussion indessen ist ihre „mediale Vermittlung [...] an eine nicht teilnehmende, aber teilhabende Zuhörerschaft" (LAUX 1995:55). Eine Fernsehdiskussion ist demnach als dialogische Kommunikation dreier Akteure mit einem inneren und einem äußeren Adressatenkreis zu verstehen: Den inneren bilden die diskutierenden Politiker samt Moderator, den äußeren indessen das Studiopublikum und die Zuschauer vor den Bildschirmen (LUGINBÜHL 1999:16f.). Die Äußerungen der Gesprächsteilnehmer 
sind an beide Kommunikationskreise gerichtet (LUGINBÜHL 1999:17), wobei jedoch eine starke Orientierung der Interaktanten an den Erwartungen des Publikums als potentieller Wähler anzunehmen ist. Diese Mehrfachadressierung der Äußerungen ist „als konstitutiv für den Gesprächsablauf“ (LAUX 1995:55) zu betrachten. Für die untersuchte Sendung ist zudem charakteristisch, dass sie live ausgestrahlt wird. Wirkliche Spontaneität ist zwar nicht möglich, weil die Gäste, besonders die Moderatorin, vorbereitet sind, dennoch sollte die Wirkung dieses medienspezifischen Faktors nicht unterschätzt werden, da er eine gewisse Unkalkulierbarkeit bedingt und somit Überraschungen in sich birgt (LUGINBÜHL 1999:24).

Für die vorgenommene Untersuchung der Sendungsausschnitte ist die Gesprächsanalyse mit ihrem Modell des Sprecherwechsels ausschlaggebend. Als grundlegende strukturelle Einheit gilt dabei der Redezug eines Sprechers (,turn'), der ,,von der Übernahme des Rederechts durch einen Sprecher bis zur Übergabe an den folgenden reicht" (SCHLOBINSKI 1996:208). Nach den Regeln der Gesprächskooperation sollte einer der Gesprächspartner das Rederecht nur an sogenannten übergaberelevanten Stellen (,transition relevance point") übernehmen, die meist „durch Phrasen- oder Satzgrenzen gekennzeichnet [sind], wobei häufig Intonationsstrukturen, Pausen oder bestimmte Partikeln eine Rolle spielen“" (SCHLOBINSKI 1996:208). Die Übernahme des Rederechts erfolgt entweder dann, wenn ein Teilnehmer das Wort erhält (,Fremdwahl ${ }^{\circ}$ ) oder wenn er sich selbst das Wort nimmt (,Selbstwahl ${ }^{\circ}$ ). Da die Fremdwahl potentiell nicht zu Konflikten führt, bleibt sie in der weiteren Darlegung unberücksichtigt. Die Selbstwahl indessen muss nicht notwendig an einer übergaberelevanten Stelle erfolgen. Versucht man also, das Wort an einer anderen Stelle zu ergreifen, kommt es zu sog. Interventionen, die nach KoTTHOFF (1993:165-174, zit. nach LUGINBÜHL 1999:94) wie folgt zu klassifizieren sind:

1. Rezipienzsignale,

2. Überlappung (Simultansequenz ohne Eingriff in das Rederecht von anderen),

3. Unterbrechung (Eingriff in das Rederecht),

3.1. reaktive Unterbrechung (früher unterbrochene Person holt sich ihr Rederecht zurück),

3.2. Expansionsverhinderung (Unterbrechung eines angekündigten längeren Gesprächsschrittes an einer transitionsrelevanten Stelle),

3.3. kompetitive Unterbrechung (markiert lokale, dringliche Konkurrenz),

4. Unterbrechungsversuch,

5. Einwurf (nicht gesprächsschrittbeanspruchende Signale, die mehr als Rezipienz signalisieren). 
Antagonistisch und daher potentiell Aggression auslösend sind vor allem kompetitive Unterbrechungen und Einwürfe, auf die in der Analyse detaillierter eingegangen wird. In Anlehnung an das oben beschriebene System des Sprecherwechsels wird bei der Analyse also zunächst unterschieden, welche kommunikative Rolle der Interaktant zum gegebenen Verlaufspunkt hat und ob er in dieser Rolle einen Redezug ausführt oder interveniert. Die Äußerung wird dann auf zwei Ebenen analysiert: Auf nonverbaler Ebene werden einerseits prosodische Eigenschaften wie Sprechgeschwindigkeit, Stimmintensität, Intonation und Akzent, andererseits motorische Phänomene wie Mimik, Gestik und Körperhaltung des Sprechers bzw. des Hörers untersucht. Auf der verbalen Ebene indessen werden Konversationsstrategien, Illokutionstyp und Anredeverhalten sowie morphologische, syntaktische und lexikalische Besonderheiten berücksichtigt. Da sowohl die Körpersprache als auch die Prosodie „Einstellungen und den expressiv-emotionalen Zustand des Sprechers [kennzeichnen] und [...] als Mittel zur erfolgreichen Erfüllung der Absichten des Sprechers und der Anpassung der Rede an die Situation [dienen]“" (GüNTHER 1999:22), führt erst das Zusammenwirken aller Elemente der genannten Dimensionen zu aggressivem Verhalten.

Die zur Analyse ausgewählten Ausschnitte werden nach den Konventionen des Gesprächsanalytischen Transkriptionssystems (GAT) verschriftet. Neue ,turns' der Sprecher beginnen mit einer neuen Transkriptzeile. Bei der Verschriftung werden nach KEHREIN (2002:IX) folgende Symbole verwendet:

$\begin{array}{ll}\text { [ ] } & \text { Überlappungen } \\ (.) & \text { Mikropause } \\ (-),(--),(--) & \text { kurze, mittlere und längere geschätzte Pausen } \\ :,::,::: & \text { Dehnung/Längung } \\ \text { äh, öh, usw. } & \text { Verzögerungssignal } \\ ((\text { lacht })) & \text { Pauschalbeschreibung } \\ ((\text { hustet })) & \text { nonverbale Handlungen } \\ \text { <empört> } & \text { interpretierende Kommentare } \\ (\text { ) } & \text { unverständliche Passage } \\ \text { (solche) } & \text { vermuteter Wortlaut } \\ \text { AKZENT } & \text { Hervorhebungsakzent }\end{array}$

An dieser Stelle ist vorauszuschicken, dass in der Analyse aggressives Verhalten der Moderatorin unberücksichtigt bleibt. Ihre privilegierte Position im Gesprächsrahmen stellt sie außerhalb meines Forschungsinteresses, da sie ihre eventuellen aggressiven Äußerungen bzw. Unterbrechungen legitimiert. 
So darf die Moderatorin beispielsweise ihren Gast - ohne jeglichen Widerspruch seinerseits - direkt kritisieren und ihn mit einer ironischen Bemerkung zu einer sachlichen Aussage zu bringen versuchen. Ein derartiges Verhalten seitens eines Diskussionsteilnehmers würde sicher zu einer Auseinandersetzung um das Rederecht führen. Die starke Position der Moderatorin wird aber von allen Diskutanten akzeptiert. Im Folgenden wird auf einige Ausschnitte der untersuchten Sendungen genauer eingegangen, wobei zur Analyse die repräsentativsten Beispiele ausgewählt wurden.

\section{Analyse 1: Renate Schmidt vs. Bischof Walter Mixa}

Die erste Szene ist dem Programm Glaubenskrieg um Rabenmütter entnommen, das dem Problem der Kinderbetreuung angesichts fehlender Krippenplätze nachgeht. Analysiert wird hier der Redezug der ehemaligen Familienministerin Renate Schmidt (SPD), die sich auf die folgende kontroverse Aussage des damaligen katholischen Bischofs von Augsburg, Walter Mixa, bezieht: Wer mit staatlicher Förderung Mütter dazu verleite, ihre Kinder bereits kurz nach der Geburt in staatliche Obhut zu geben, degradiere die Frau zur „Gebärmaschine“.

\section{Transkriptausschnitt 1:}

Sendung: $\quad$ Glaubenskrieg um Rabenmütter vom 12.4.2007

Zeit: 11:27h -13:50h

Sprecher:

RS: Renate Schmidt

MI: Maybrit Illner

BSS: Beatrix Selk-Schnoor

1 MI: dann ganz ganz kleine sekunde (.) dann möchte ich an der stelle jetzt doch erstmal

2 renate SCHMIDT (-) ehemalige familienministerin (.) aber auch BAYERIN mit in der

3 runde begrüßen (.) und sie als nächstes und erstes fragen (.) ob es vielleicht auch so ein

4 bisschen mit ner form bayerischer DEFTIGKEIT zu tun hat (.) in welcher

5 HEFTIGKEIT wir gerade diskutieren und welche verbal' INJURIEN hier so hin und her

6 wandern 
$7 \quad$ RS: also ich mag eigentlich den bischof mixa ganz [gern

8 MI: [er sitzt links neben [mir

9 RS: [und dachte [mir

10 MI: [auch neben Ihnen

11 RS: dachte mir (.) was ist denn in den TYPEN eigentlich gefahren (--) und verehrtester

12 bischof (.) ich muss ihnen mal sagen (.) ich dachte mich trifft wirklich der SCHLAG (.)

13 als ich mich plötzlich von ihnen (.) als GEB ÄRMASCHINE titulieren lassen musste (.)

14 weil ich habe drei (.) in der zwischenzeit SEHR erwachsene kinder (.) die ICH liebe (.)

15 die MICH lieben (.) ich habe vier enkelinnen (.) also meine kinder haben sich trotz ihrer

16 UNERTRÄGLICHEN erfahrungen mit der gebärmaschine renate schmidt haben sich

17 [wieder für kinder

18 BSS: [missverständnis

19 RS: haben sich [wieder für

20 BSS: [missverständnis

21 RS: [haben sich wieder für kinder entschieden

22 BSS: missverständnis ja

23 RS: ich habe gedacht DER ist jetzt plötzlich DURCHGEDREHT ((Mixa schmunzelt))

24 und dann hab' ich gedacht (.) vielleicht beruhigt er sich wieder (.) über gewisse

25 zeiträume schien es der fa der fall zu sein (-) und jetzt plötzlich bin ich in meiner

26 WÜRDE verletzt weil ich mich entschieden habe (-) TEILS weil ich's musste (-) TEILS

27 weil ich's aber auch wollte nach dem dritten kind (-) bei den ersten zwei hätt's gar nicht

28 die möglichkeit gegeben (--) weil ich mich entschieden habe ERWERBSTÄTIG zu sein

29 (-) ich VERMEIDE das wort arbeiten (.) weil ich natürlich jeden RESPEKT auch vor

30 dem familienmodell von IHNEN habe ((wendet sich an BSS)) ist da überhaupt KEINE 
31 frage (-) und ich finde (.) wir sollten diese ABSURDE diskussion (-) auf der einen seite

32 die angeblich verantwortungslosen erwerbstätigen RABENMÜTTER und auf der

33 anderen seite die angeblich etwas depperten nur HAUSFRAUEN (-) wir sollten diese

34 ABSURDE diskussion endlich verschwinden lassen (.) und SIE sind ihrer würde als

35 BISCHOF nicht gerecht geworden <empört> (-) um's ihnen mal DEUTLICH zu sagen

36 (.) weil sie sollten frauen keine ANGST einjagen davor kinder in die welt zu setzen (.)

37 auch wenn sie sich für erwerbstätigkeit entscheiden (.) und ((Applaus des Publikums))

38 genau DAS haben sie gemacht <aufgeregt>

39 BSS: oh::: darum geht es überhaupt NICHT (.) darum geht es überhaupt NICHT

40 <empört>

Von der Moderatorin zur Meinungsäußerung aufgefordert, beginnt Schmidt ihre Äußerung mit dem Versuch, den Bischof in den Augen des Publikums herabzusetzen. Mit dem kontrastierenden ich mag eigentlich den bischof mixa ganz gern [7]; dachte mir was ist denn in den TYPEN eigentlich gefahren [11] betont sie, dass sie ihn eher als eine Privatperson, denn als einen Vertreter der katholischen Kirche betrachtet. Mit der Anrede in der 3. Person (ich mag eigentlich den bischof [7]; vielleicht beruhigt er sich wieder [24]) schafft sie Distanz und klammert den Bischof aus der Diskussion aus. Diese Herabwürdigung wird zusätzlich auf morphologischer Ebene mit den im Satz statt Personalpronomina akzentuierend verwendeten Demonstrativpronomina und auf lexikalischer Ebene mit despektierlichen umgangssprachlichen Verben, Nomina und Wendungen verstärkt (DER ist jetzt plötzlich DURCHGEDREHT [23]; was ist denn in den TYPEN eigentlich gefahren [11]; salopp mich trifft wirklich der SCHLAG [12]). Diese sprachliche Diskreditierung des Bischofs wird von der Moderatorin sofort als, gesichtsbedrohender Akt" (,face threatening act“, vgl. BROWN / LEVINSON 1987:60) qualifiziert. Sie versucht daher mit dem Einwurf er sitzt links neben mir auch neben ihnen [8] dieses distanzierende Nicht-Anreden sofort zu monieren. Die Bemerkung der Moderatorin nimmt Schmidt mit einem Kopfnicken zur Kenntnis, das zeigen soll, wie bewusst sie sich für diese Strategie entschieden hat, die auf non- 
Agnieszka Poźlewicz

verbaler Ebene zusätzlich durch Blickkontakt mit dem Bischof und Hinwendung des Kopfes zu ihm konterkariert wird.

Die indirekte Kritik geht in einen direkten Appell über (sie sollten frauen keine ANGST einjagen davor kinder in die welt zu setzen [36]) und in einen direkten Vorwurf (SIE sind ihrer würde als BISCHOF nicht gerecht geworden [34]; genau DAS haben sie gemacht [38]), Schmidt wechselt ihr Anredeverhalten und spricht ihren Mitstreiter direkt an, an einer Stelle sogar mit Titel samt elativem Adjektiv als verehrtester bischof [11]. Die gesamte Äußerung ist von Gefühlen wie Empörung und Ärger geprägt, was sich auch in der energischen Körpersprache (stakkatohafte Auf- und Abbewegungen der Schlaghand, Kopfnicken als Bekräftigung der akzentuierten Stellen, Streckhand zur Verstärkung des Vorwurfs) der Disputantin widerspiegelt. Der emotive Charakter der Aussage wird auch an der Prosodie sichtbar: Schmidt spricht immer schneller und lauter, was besonders bei den Einwürfen seitens der Vertreterin des Familiennetzwerks Deutschland Beatrix SelkSchnoor zu beobachten ist. Erhöhte Sprechgeschwindigkeit und Stimmstärke werden erfolgreich eingesetzt, um das Rederecht zu erhalten oder durchzusetzen.

Die analysierte Äußerung markiert auch die mit temporalem und dynamischem Akzent auf den Namen der Schlüsselbegriffe verstärkte Ironie. Die Anspielung auf die Worte des Bischofs (gebärmaschine renate schmidt [16]) sowie die ad absurdum geführten Bemerkungen über die eigene Mutterschaft (meine kinder haben sich trotz ihrer UNERTRÄGLICHEN erfahrungen [...] für kinder entschieden [15]), hervorgehoben durch besondere Betonung, bringen Schmidts Empörung deutlicher zum Ausdruck als ihre direkte Feststellung plötzlich bin in meiner WÜRDE verletzt [25].

Der Vorwurf, dass die bisher geführte Diskussion absurd sei, wird einerseits durch eine Wir-Botschaft gemildert (wir sollten diese ABSURDE diskussion endlich verschwinden lassen [33]), die Zusammengehörigkeit und in gewissem Sinne die Mitverantwortung der Diskutanten betont. Andererseits aber unterstellt Schmidt implizit ihren Opponenten, sich nicht von stereotypem Denken befreien zu können, indem sie ihnen polarisierende Argumentation vorwirft (die angeblich verantwortungslosen erwerbstätigen RABENMÜTTER [32] vs. die angeblich etwas depperten nur HAUSFRAUEN [33]). Die hier antonymisch gesetzten nominalen Zusammensetzungen rufen durch pejorative Adjektive verstärkte negative Assoziationen hervor. 


\section{Analyse 2: Renate Schmidt vs. Beatrix Selk-Schnoor}

Die Auseinandersetzung zwischen Schmidt und Selk-Schnoor wird in der zweiten von uns analysierten Szene fortgesetzt. Sichtbar wird dabei der Mechanismus des Sprecherwechsels an mehreren Einwürfen und Unterbrechungen.

\section{Transkriptausschnitt 2:}

Sendung: $\quad$ Glaubenskrieg um Rabenmütter vom 12.4.2007

Zeit: $\quad$ 18:00h - 20:25h

Sprecher: $\quad$ RS: Renate Schmidt

MI: Maybrit Illner

BSS: Beatrix Selk-Schnoor

UW: Ulrich Wickert

1 BSS: es haben VIERZIG prozent der frauen gesagt (-) ich möchte mein kind ERST

2 zwischen dem DRITTEN und dem VIERTEN lebensjahr in die krippe geben $(--)$

3 in einen kindergarten eigentlich dann schon (--) und noch mal DREISSIG prozent würden

4 sogar WARTEN bis zur einschulung des kindes (-) bis zum SIEBTEN lebensjahr (--)

5 SO sind die verhältnisse (-) es sind BETROFFENE gefragt (-) und NICHT wie bei

6 allensbach querbeet (--) betroff[ene gefragt

7 MI: [frau schnoor

8 BSS: junge mütter

9 RS: die realitäten sind vollkommen ANDERS <aufgeregt>

10 BSS: die REALITÄTEN sind (.) dass die frauen keine WAHL haben (.) sondern

11 arbeiten gehen müssen (.) weil die familien FÜNFZIG prozent weniger einkommen

12 haben (.) als die vergleichbaren KINDERLOSEN (---) das ist die REALITÄT in

13 deutschland (-) das ist die [REALITÄT

14 RS: [noch mal sie können sich keine einzige ((wird unterbrochen)) 
Agnieszka Poźlewicz

BSS: und ich BITTE sie (-) wir haben eine ERBÄRMLICHE diskussion erlebt in der letzten zeit (-) wir haben nicht mehr ZUHÖREN können (-) nicht mehr UNTERSCHEIDEN können (---) bischof mixa hat NIE gesagt (-) dass frauen gebärmaschinen sind (--) hören sie BITTE mal alle RICHTIG gut zu (--)er hat gesagt(-) die POLITISCHEN verhältnisse sind SOLCHE dass frauen zu gebärmaschinen

degradiert werden (--) ein vorwurf den vor einigen [jahren germaine GREER

UW: [somit hat er ja das GESAGT

BSS: die altfeministin [schon gesagt hat

UW: [damit hat er das GESAGT

(Unverständliche Passage)

MI: [sekunde (--) mein VORSCHLAG (-) frau SCHMIDT ist dran (-) dann ist der

BISCHOF dran

RS: es gibt keinerlei VERPFLICHTUNG (.) ein kind in eine krippe [zu schicken

BSS: [DOCH (.) finanzielle (--) nöte (--) [nöte

RS: [oder in eine kindertagesstätte (-) es sind bei uns heute in den kindergärten (--)

ich rede GAR nicht über krippen (-) sind also auch die kinder von frauen (.) die ZU HAUSE

sind

BSS: kindergärten WUNDERBAR

RS: in kindergärten sind von frauen (.) also es gibt was sie hier erzählen (.) mit IHREN

vierzig prozent (.) und dass vierzig dreißig prozent ihre kinder bis zum SCHULALTER

zu hause lassen würden (-) das stimmt NICHT [(-) es ist

BSS: [das STIMMT

RS: es ist schlicht und einfach NICHT WAHR

BSS: dann BESTREITEN sie die meinungsumfragen [von leuten

RS: [ich spreche von REALITÄTEN (-) ich spreche NICHT von umfragen (.) sondern

von FAKTEN (-) über NEUNZIG prozent der kinder gehen im letzten jahr vor der 
41 schule in die [kindergärten

42 BSS: [wunderbar wunderbar (.) jawohl (.) ist [alles in ordnung

43 RS: [es gibt SIEBZIG prozent der kinder in den kindergärten zwischen dem VIERTEN

44 und dem FÜNFTEN lebensjahr (---) und wir wissen in den LÄNDERN (--) in den

45 bundesländern in deutschland

46 BSS: die frau hat ja unter umständen ihr NÄCHSTES kind bekommen und das DRITTE

47 und VIERTE

48 RS: darf man (.) darf man bei ihnen eigentlich IRGENDWANN einen satz zu ende bringen <aufgeregt>

BSS: JA

RS: das ist REIZEND ((ironisches Lächeln)) (--) ja (.) also ich möchte hier keinen

52 ZICKENKRIEG machen (.) sondern vielleicht können SIE mal zuhören und ich höre

53 IHNEN gerne zu (.) und dann wird was VERNÜNFTIGES [daraus (.) ja

54 BSS: [ICH habe lange [zugehört

55 RS: [WUNDERBAR wunderbar (-) ja (.) wir haben ihnen AUCH sehr lange zugehört

$56 \quad$ ((Applaus des Publikums))

In ihrer Erwiderung bezieht sich Schnoor auf Umfragen, um ihre Argumentation zusätzlich zu stützen. Deutlich ist an dieser Stelle ihr Versuch, mit der Bemerkung es sind BETROFFENE gefragt und NICHT wie bei allensbach querbeet [5] Ergebnisse anderer Umfragen zu diskreditieren. Den neutralen Charakter dieser Sequenz bestimmen gemäßigtes Sprechtempo, neutrale Intonation und - auf nonverbaler Ebene - leichte Handbewegungen wie Falthände, Takthand, Spitzhand und Wandhände, die ihre Argumentation stützen. Der Unterbrechungsversuch seitens der Moderatorin (leise direkte Anrede frau schnoor [7]) wird von Schmidt als Zeichen einer übergaberelevanten Stelle interpretiert. Es kommt zur Expansionsverhinderung (die realitäten sind vollkommen ANDERS [9]), die jedoch teils wegen der steigenden Intonation von Schnoor als direkte Unterstellung von Inkompetenz und verbaler Gewalt empfunden wird. Um das eigene Rederecht $\mathrm{zu}$ verteidigen, übernimmt Schnoor die von der Rivalin verwendete Struktur (die REALITÄTEN sind dass die frauen keine WAHL haben sondern arbeiten gehen müssen [10]; 
Agnieszka Poźlewicz

das ist die REALITÄT in deutschland [12]), die sie mehrmals wiederholt. Ihre Irritation spiegelt sich einerseits in überhöhter Sprechstimmlage, erhöhter Stimmstärke und Sprechgeschwindigkeit, andererseits aber in immer energischer werdender Gestikulation wider. Zur Bekräftigung ihrer Aussage bedient sich Schnoor ihres in Richtung Schmidt gestreckten Zeigefingers (einmal sogar beider Hände). Den moralisierenden Zeigefinger erhebt sie auch bei der emphatisch gesprochenen Aufforderung ich BITTE sie [15] und hören sie BITTE mal alle RICHTIG gut zu [18], die als gesichtsbedrohender Akt gewertet werden kann. Selk-Schnoor wirft den Streitern vor, dass sie die Worte des Bischofs absichtlich falsch interpretieren (bischof mixa hat NIE gesagt dass frauen gebärmaschinen sind [17]), was auf prosodischer Ebene durch melodischen Wortakzent und steigende Intonation unterstützt wird. Darüber hinaus beruft sich Schnoor auf eine ähnliche Aussage von Germaine Greer, einer Ikone der Frauenbewegung, um ihrer Argumentation besonderes Gewicht zu verleihen. Die hier angewandte typische Argumentationsstrategie ist wirksam, weil die zitierte Autorität eigentlich auf der Seite der Opponenten liegt.

Als das Rederecht an Schmidt übergeht, versucht Schnoor, die Rivalin mit besonders unhöflichen - weil die Gesprächspartnerin demütigenden - Korrekturen ihrer Aussagen, sarkastischen Bemerkungen (wunderbar wunderbar jawohl ist alles in ordnung [42]) sowie mit der Verteidigung eigener Argumente zu stören; u.a. wiederholt sie mit Nachdruck das STIMMT [36]. Die Einwürfe sind jedoch nicht Eingriffe in das Rederecht, sondern eher Signale negativer Emotionen wie Empörung, Irritation, Verärgerung, die auch steigende Intonation kennzeichnet.

Schmidt indessen versucht, nach Erhalt des Rederechts von der Moderatorin, die bisherige Aussage ihrer Opponentin zu diskreditieren. Einerseits bedient sie sich dazu direkter Unterstellung von Unehrlichkeit und Inkompetenz, die sie zusätzlich durch Verwendung der umgangssprachlichen phraseologischen Wendung es ist schlicht und einfach NICHT WAHR [37]; das stimmt NICHT [35] verstärkt und prosodisch mit erhöhter Stimmstärke und dynamischem Akzent artikuliert. Andererseits bedient sich Schmidt der Polarisierung (Fakten vs. Umfragen). Um die Argumentation der Opponentin zu delegitimieren, steht hier auch das stigmatisierende Possessivum (mit IHREN vierzig Prozent [33]), mit denen Schmidt die Aussagekraft der zitierten statistischen Daten bagatellisiert.

Der Kampf um das Rederecht führt zur gesichtsbedrohenden ironischen Frage darf man bei ihnen eigentlich IRGENDWANN einen satz zu ende brin- 
gen [48]; das ist REIZEND [51]. Diese ist ein Versuch, die störenden Einwürfe und Unterbrechungen zu kritisieren oder abzuwehren. Die sarkastische Äußerung ist zudem von einem herablassend-vergebenden Lächeln begleitet. Die Frage mit dem verallgemeinernden Indefinitpronomen man geht über in die direkt adressierte Äußerung ich möchte hier keinen ZICKENKRIEG machen [51]; vielleicht können SIE mal zuhören [52]. Die nominale Zusammensetzung Zickenkrieg zeigt einerseits die aggressive Einstellung gegenüber der Opponentin (Krieg) und ist andererseits eine indirekte Beleidigung (Zicke). Die verbal so angegriffene Schnoor versucht, ihr Gesicht zu wahren, indem sie der Opponentin direkt erwidert: ICH habe lange zugehört [54]. Sie bleibt jedoch verunsichert, was ihr Gesichtsausdruck und ihre verhaltene Stimmstärke anzeigen.

\section{Analyse 3: Sigmar Gabriel vs. Wolfram Weimer}

Der folgende Ausschnitt ist der Sendung Strom, Sprit, Steuern entnommen, in der das Problem des Energiepreisanstiegs im Zusammenhang mit dem Umweltschutz erörtert wird. Es kommt zu einer regen Auseinandersetzung zwischen dem SPD-Umweltminister Sigmar Gabriel und dem Journalisten Wolfram Weimer, der als einziger in der Runde vor Hysterie in der Debatte warnt und die Schuld des Menschen am Klimawandel bezweifelt.

\section{Transkriptausschnitt 3:}

Sendung: $\quad$ Strom, Sprit, Steuern vom 5.7.2007

Zeit: $\quad 21: 55 \mathrm{~h}-23: 50 \mathrm{~h}$

Sprecher: $\quad$ SG: Sigmar Gabriel

WW: Wolfram Weimer

MI: Maybrit Illner

1 WW: ich glaube übrigens (.) dass man NICHT das große KLIMATHEMA in hundert

2 jahren braucht (.) um die gesellschaft ENERGIEEFFIZIENTER umzubauen (--) wir

3 haben ganz KONKRET politische risiken (-) auf die wir eingehen (---) wir machen uns

4 abhängig von HALBDESPOTEN in russland (.) und von ganzen DESPOTEN in arabien 

interesse (-) und DAZU

3 SG: nö: nö:: es ist genau (--) JETZT haben wir (-) sind SIE endlich mal DA (-) wo sie

14 sich mal BEGRÜNDEN müssen (--) denn es ist eben FALSCH (-) was sie behaupten (-)

15 dass die CO2-konzentration seit JAHRMILLIONEN irgendwie sozusagen immer um

16 eine linie pendeln würde (-) sondern seit 1980 hat sie (.) wieder das letzte mal in

17 karbonzeitalter (.) und da sah die erde WIRKLICH etwas anders aus (-) DRAMATISCH

18 zugenommen (--) wir haben tausend achthundert WISSENSCHAFTLER dieser welt (.)

19 die sagen (-) es ist NICHT apokalyptisch (-) sondern das ist GEFÄHRLICH (.) für viele

20 menschen auf der ERDE (--) sie werden mit ihrem LEBEN dafür büßen müssen

21 MI: ok

22 SG: und was SIE machen ist (--) sie zitieren ZEHN prozent der wissenschaftler (-) die

23 alle KEINE klimaforscher sind (-) alle KEINE klimaforscher (-) das ist (-) und sagen (-)

24 na ja (.) eigentlich müsste man es nicht so fürchterlich ERNST nehmen (--) sie

RELATIVIEREN das Problem (--) wissen sie wie das ist (-) als ob jemand sagt (--) na ja

(-) zu NEUNZIG prozent wird das flugzeug ABSTÜRZEN (-) aber es gibt doch ZEHN LANDET 
28 irgendwo (-) und da sage ich ihnen ganz OFFEN (-) da können WIR nicht mitmachen (-)

29 da werden WIR uns schon darauf verlassen müssen (.) dass da GEFÄHRLICHE

30 entwicklungen passieren (-) mal jetzt ohne apokalyptisch zu sein (--) wer

31 APOKALYPTISCH ist (.) kann keine lösung produzieren (-) aber was SIE tun ist (.) sie

32 RELATIVIEREN das problem (-) und ich finde es gar GUT (.) dass ihnen

33 RAUSGERUTSCHT ist dass sie sagen (.) das mit dem klima ist seit

34 JAHRMILLIONEN so (-) exakt DAS ist FALSCH was sie eben behauptet [haben

35 WW: [herr gabriel (-) es ist mir gar nicht ausgerutscht (.) sondern ich SEHE es so

36 <enttäuscht> (--) natürlich ist das klima per definitionem WANDEL unterlegen (.) und

37 ihr EIGENES institut für [geoforschung

38 SG: [ist nicht MEINS

39 WW: nicht IHRES aber

40 SG: das sind auch KEINE klimaforscher (.) das WISSEN sie auch

41 WW: es ist ein HOHES expertengremium

42 SG: NEIN ist es nicht

43 WW: DOCH ist es schon (--) und sie müssen doch nicht leute die ANDERER meinung sind so ABBÜGELN

45 SG: MACH' ich nicht, [sage ich nur

46 WW: [DOCH tun [sie

47 SG: [sage ich nur ((wird unterbrochen))

48 WW: lassen sie mich doch

49 MI: lassen sie ARGUMENTIEREN

Auslöser des Streitgespräches ist die kontroverse Feststellung von Weimer, man brauche keine große Klimageschichte [7], um die Umwandlung der Energieversorgung zu rechtfertigen. An dieser Stelle ergreift Gabriel das Wort, ohne jedoch die Aufforderung der Moderatorin (herr gabriel sekunde [10]) zu beachten. Mit seinem Ausruf (aber es gibt SIE [9]; es gibt diese GROSSE klimageschichte [11]), bei dem er die vom Gegner verwendete Nominalphrase wiederholt, stellt er Weimers Argumentation infrage und wirft ihm Inkompetenz vor. Auf den Einwurf des Opponenten (klimawandel 
Agnieszka Poźlewicz

tut sich seit JAHRMILLIONEN [12]) reagiert er mit lauter Verneinung (nö nö [13]), und bekräftigt diese mit der Bewegung der Halt-Hand. Danach diskreditiert er die bisherigen Argumente seines Opponenten, indem er sie explizit als falsch bezeichnet (es ist eben FALSCH was sie behaupten [14]). Seine Absicht, den Opponenten herabzusetzen, kommt noch stärker durch die sarkastische Bemerkung über das Klima im Karbonzeitalter zum Ausdruck (da sah die erde WIRKLICH etwas anders aus [17]), die er mit einem ironischen Lächeln verstärkt. Im Weiteren kritisiert Gabriel seinen Gesprächspartner, indem er ihn belehrend darauf aufmerksam macht, dass er sich in seinen Argumenten auf inkompetente Autoritäten beruft. Er wiederholt den gesichtsbedrohenden direkten Vorwurf, Weimer relativiere das Problem. Um diese Unterstellung zu verdeutlichen, bedient er sich des Beispiels eines Flugzeugsabsturzes. Auf nonverbaler Ebene bekräftigt er seine Aussage durch ein ironisches Grinsen und die Bewegungen seines in Richtung Weimers gestreckten Zeigefingers. Eine weitere Strategie, der sich Gabriel bedient, um seinen Mitstreiter zu diskreditieren, ist die Wir-Botschaft, die signalisieren soll, er sei nicht der einzige, der die Argumentation des Opponenten für absurd hält. So kommt es zur Polarisierung der Gegner versus wir (ich und die, für die ich spreche).

Um die Vorwürfe des Opponenten abzuwehren und sein Gesicht zu wahren, beruft sich Weimer auf das Institut für Geo-Forschung, das jedoch sofort von Gabriel als KEINE klimaforscher [40] und kein HOHES Expertengremium [WW 41; SG 42] abgelehnt wird. Es kommt an dieser Stelle zu einem unangenehmen Schlagabtausch, wobei die Einwürfe und Unterbrechungsversuche beider Seiten der Unterstellung von Inkompetenz dienen sollen. Im Endeffekt fühlt sich Weimer verbal angegriffen, was er auch seinem Gesprächspartner in Form eines allgemeinen Ratschlags mit dem Modalverb müssen vorwirft (sie müssen doch nicht leute die ANDERER meinung sind so $A B B \ddot{U} G E L N$ [43]). Die steigende Intonation und erhöhte Stimmstärke sind dabei Ausdruck negativer Emotionen. Erst nach der Intervention der Moderatorin, die Gabriel ermahnt (lassen sie ARGUMENTIEREN [49]), ist ein sachliches Gespräch wieder möglich. Noch einmal wird an dieser Stelle die besondere Position der Moderatorin deutlich, die den sachlichen Ablauf der Diskussion zu sichern hat. 


\section{Analyse 4: Günther Beckstein vs. Heiner Geißler}

Der folgende Ausschnitt aus der Sendung Razzien und Randale thematisiert die von der Polizei auf der einen Seite und von den Demonstranten auf der anderen vorgenommenen Vorbereitungen auf den G8-Gipfel. In ihm kommt es zu einer Auseinandersetzung zwischen dem Bayerischen CSU-Innenminister Günther Beckstein und dem ehemaligen CDU-Generalsekretär Heiner Geißler. Den regen Schlagabtausch löst Heiner Geißler aus, der eine kurz zuvor von der Polizei durchgeführte Großrazzia kritisiert und seinem Opponenten vorwirft, dass das Innenministerium dafür verantwortlich ist.

\section{Transkriptausschnitt 4:}

Sendung: $\quad$ Razzien und Randale vom 31.5.2007

Zeit: $\quad 15: 38 \mathrm{~h}-16: 45 \mathrm{~h}$

Sprecher: GB: Günther Beckstein

HG: Heiner Geißler

MI: Maybrit Illner

1 MI: bevor herr beckstein noch einmal dran ist ((wird von Beckstein unterbrochen))

2 GB: weil die BUNDESANWALTSCHAFT einen durchsuchungsbefehl (unverständliche Passage)

HG: sehen sie (.) JETZT kommt nämlich ((wird unterbrochen))

4 GB: die BUNDESANWALTSCHAFT und zwar (.) wegen des VERGEHENS einer

5 schweren BRANDSTIFTUNG und die justiz ist [unabhängig

6 HG: [diese vierzig LEUTE (.) die da untersucht worden sind (.) die haben doch keine

7 BRANDSTIFTUNG [begangen <empört>

8 GB: [entschuldigung aber die BUNDESANWALTSCHAFT war der meinung (.) dass es

9 schwere verdachte sind (unverständliche Passage)

10 HG: Ja (.) jetzt erregen sich die INNENMINISTER (-) sie reden sich bei der großrazzia

11 jetzt HERAUS (-) das seien gar nicht SIE gewesen (.) sondern das sei die

12 BUNDESANWALTSCHAFT [gewesen

13 GB: [natürlich [nicht 
HG: [ja weiß man eigentlich in DEUTSCHLAND (.) die innenminister (-) wissen die

16 GB: entschuldigung (--) wir haben ne UNABHÄNGIGE justiz herr geißler <aufgeregt>

17 HG: das ist doch INDISKUTABEL <empört>

18 GB: entschuldigen sie (.) wenn ich mal sage (.) ich REG' mich ehrlich gesagt AUF (-)

19 dass JEMAND (.) der ein verantwortlicher MINISTER war jetzt DAHERREDET (.)

obwohl er weiß

HG: ICH red' NICHT [daher ((erhöhte Stimmstärke))

GB: [ER redet so DAHER obwohl er weiß (.) dass die bundesanwaltschaft eine

23 UNABHÄNGIGE einrichtung ist (.) und dass die GERICHTE eine durchsuchung

24 angeordnet haben (.) und das wird MIR jetzt in die schuhe [geschoben

25 HG: [nein

26 GB: das ist nicht in ORDNUNG <empört>

27 HG: überhaupt nicht

28 GB: sondern das war die unabhängige JUSTIZ die schwere STRAFTATEN aufzuklären

29 hatte (.) vielleicht ist das richtig oder falsch (.) aber den EINDRUCK zu erwecken (.)

30 das sei POLIZEIWILLKÜRLICH (.) das halte ich für SKANDALÖS (.) insbesondere

31 von JEMANDEM der selber VERANTWORTUNG getragen hat ((erhöhte

32 Sprechgeschwindigkeit))

Der Versuch der Moderatorin, auch andere in die Diskussion einzubeziehen, (bevor herr beckstein noch einmal dran ist [1]) wird von Beckstein ignoriert, um das Rederecht zu behalten. Um die Vorwürfe des Opponenten zurückzuweisen, wiederholt er nachdrücklich die für seine Argumentation wichtigen Schlüsselwörter (Bundesanwaltschaft, Durchsuchungen, Verdacht, Brandstiftung), die er besonders akzentuiert. Seine Empörung und Aufregung drücken sich auch in seiner hohen Sprechgeschwindigkeit aus.

Geißler indessen versucht, seinen Gegner zu unterbrechen und das Rederecht zu übernehmen, indem er sich direkt an Beckstein wendet (sehen sie [4]) und 
seine Argumente in Frage stellt (diese vierzig Leute [...] die haben doch keine BRANDSTIFTUNG begangen [7]). Die Abtönungspartikel doch ist in diesem Fall Signal von Entrüstung und Unmut. Der Unterbrechungsversuch, der erst beim zweiten Mal zur Übernahme des Rederechts führt, wird auf nonverbaler Ebene durch den in Richtung des Opponenten gestreckten Zeigefinger und Blickkontakt gestützt. Den allgemein formulierten Vorwurf (Innenminister [...] reden sich bei der großrazzia jetzt HERAUS [11]) richtet Geißler eigentlich an seinen Gegner, wobei diese Indirektheit den damit verbundenen gesichtsbedrohenden Akt mildern sollte. Der Vorwurf geht dann in eine ironische Bemerkung über (die innenminister wissen die nicht mehr was die BUNDESANWALTSCHAFT eigentlich tut [15]), auf die Beckstein sofort mit einer belehrenden und somit herabwürdigenden Aussage (wir haben ne UNABHÄNGIGE justiz herr geißler [17]) reagiert. Diese wird von Geißler als Beleidigung empfunden und mit dem Ausruf das ist doch INDISKUTABEL [18] abgewehrt. Seine Empörung zeigt einerseits die steigende Intonation, andererseits die Bewegung der Spreizhand in der Höhe der Stirn, was die Absurdität von Becksteins Bemerkung unterstreichen soll. Auf verbaler Ebene indessen wird die Entrüstung durch die Abtönungspartikel doch bekräftigt.

Das Rederecht bleibt jedoch bei Beckstein, der in seinem ,turn' dem Opponenten Inkompetenz unterstellt, wobei er sich allerdings nicht direkt an Geißler wendet, sondern sich der Umschreibung in der 3. Person bedient (ich REG' mich [...] AUF dass JEMAND der ein verantwortlicher MINISTER war jetzt DAHERREDET [19]). Die Beleidigung, mit dem negativ konnotierten Verb daherreden formuliert, wird durch die Verwendung der 3. Person deutlich abgemildert. Nichtsdestotrotz fühlt sich Geißler angesprochen und reagiert mit einem Ausruf der Empörung (ICH red 'NICHT daher [22]), der auf nonverbaler Ebene mit Kopfschütteln, warnendem Zeigefinger und dem Blickkontakt mit dem Publikum bekräftigt wird. Beckstein setzt seine Kritik an Geißler fort, indem er seine bisherigen Äußerungen negativ bewertet (das ist nicht in ORDNUNG [27]; das halte ich für SKANDALÖS [31]). Charakteristisch für seinen turn sind die steigende Intonation, erhöhte Stimmstärke und besondere Akzentuierung der negativ konnotierten Wörter. Auch die stakkatohaften Bewegungen der Streckhand und das Vermeiden des Blickkontaktes mit seinem Opponenten betonen die negativen Emotionen des Sprechers. Seinen Redezug beendet Beckstein mit der impliziten Unterstellung, sein Gegner sei unaufrichtig (das halte ich für SKANDALÖS insbesondere von JEMANDEM der selber VERANTWORTUNG getragen hat [31]), die wieder durch die Äußerung in der 3. Person erreicht wird. Um zu zeigen, 
dass er seinen ,turn' beendet hat, aber keine Gegenargumente hören will, wendet er Geißler den Rücken zu. Der weitere Meinungsaustausch wird von der Moderatorin unterbrochen, die sich an dieser Stelle mit einer Frage an ihren nächsten Gast wendet.

Aus der Analyse geht hervor, dass sprachliche Aggression ein äußerst komplexes Phänomen ist. Auf verbaler Ebene neutrale Äußerungen können zu besonders aggressiven, das Gesicht des Gesprächspartners bedrohenden Aussagen werden, wenn sie mit entsprechenden nonverbalen Elementen kombiniert sind. So kann beispielsweise ein ironisches Grinsen den Ausdruck der Missachtung bekräftigen und eine ausgestreckte Halt-Hand den Versuch, den Gesprächspartner zu unterbrechen, verdeutlichen. Anhand der bisherigen Untersuchungen kann angenommen werden, dass $\mathrm{zu}$ besonders aggressiven Gesten Schlaghand, Streckhand und Zeigefinger, den man als Verlängerung oder Spezifikation der Streckhand verstehen kann, zu rechnen sind. Die in Anlehnung an WEINRICH (1992) genannten Gesten können Ausdruck des Machtanspruchs und der Dominanz des Sprechers über seinen Gesprächspartner sein. Darüber hinaus bewirkt der Zeigefinger eine zusätzliche Stigmatisierung der mit ihm identifizierten Person und hat so ausgrenzende Funktion (MüLLER 1996:202-205). Dazu kommen prosodische Elemente wie Intonation oder Akzentuierung als Ausdruck negativer Emotionen. Zudem ermöglicht erhöhtes Sprechtempo einhergehend mit steigender Stimmstärke, den Opponenten während der Diskussion zu übertönen, was zugleich die eigene Überheblichkeit vorführt.

Die Spezifik politischer Fernsehdiskussionen beruht darauf, dass in ihnen Argumentieren und Propagieren verflochten sind bzw. werden: Man wirbt diskret für die eigene Person bzw. Partei und versucht zugleich, seinen Gegner und dessen Position abzuwerten, indem man ihm beispielsweise Inkompetenz oder Unaufrichtigkeit und negative Absichten oder Eigenschaften unterstellt (LUGINBÜHL 1999:41f., 214-216). Anhand des analysierten Materials kann davon ausgegangen werden, dass Politiker bemüht sind, ihr aggressives Verhalten eher zu verschleiern als zu exponieren. Da sich Politiker immer an ihren potentiellen Wählern orientieren, bedienen sie sich impliziter Mittel wie ironischer Bemerkungen bzw. Unterstellungen, um nicht oder nur begrenzt für die eigenen Worte verantwortlich zu sein. Diesen Unterschied im Verhalten von Politikern und von ,Otto Normalverbraucher" mag folgendes Beispiel veranschaulichen: Während ein Politiker seinem Mitstreiter vorwirft, seine Argumentation sei Betreibung wissenschaftlicher Rabulistik (Sigmar Gabriel zu Wolfram Weiner in der Sendung Strom, Sprit, Steuern 
vom 5.7.2007), ist es einer zur Diskussion um Lohn-Dumping als Betroffene eingeladenen Niedriglöhnerin erlaubt, die Aussage des Gesprächspartners mit das ist doch Quatsch oder ganz derb das ist mir scheißegal zu kommentieren (Susanne Neumann in der Sendung Billig, befristet, bedroht vom 17.5.2007).

\section{Literatur}

Brown, Penelope / Levinson, Steven (1987): Politeness. Some universals in language usage. Cambridge.

GÜNTHER, CARSTEN (1999): Prosodie und Sprachproduktion. Tübingen.

HoffMann, Rolf-RÜDIGER (1982): Politische Fernsehinterviews. Eine empirische Analyse sprachlichen Handelns. Tübingen.

HundSNURSCHER, Franz (1994): Dialog-Typologie. In: FritZ, GERD / HundSNuRSCHER, FRANZ (eds.): Handbuch der Dialoganalyse. Tübingen, 203-238.

KeHreIn, Roland (2002): Prosodie und Emotionen. Tübingen.

KotTHOFF, HelGA (1993): Unterbrechungen, Überlappungen und andere Interventionen. Vorschläge zur Kategorienunterscheidung und kontextorientierten Interpretation. In: Deutsche Sprache 21:162-185.

LAUX, HeIKE (1995): Verbale Aggression am Beispiel einer politischen Fernsehdiskussion. Wirkungsanalyse eines Streitgespräches. Stuttgart.

LUGINBÜHL, MARTIN (1999): Gewalt im Gespräch. Verbale Gewalt in politischen Fernsehdiskussionen am Beispiel der „Arena“. Bern/Berlin/Frankfurt (M.)/New York/Paris/Wien.

MÜLlER, CORNELIA (1996): Zur Unhöflichkeit von Zeigegesten. In: HAFERLAND, HARALD / PAUL, INGWER (eds.): Höflichkeit. Oldenburg (=Osnabrücker Beiträge zur Sprachtheorie 52), 196-222.

SCHLOBINSKI, PETER (1996): Empirische Sprachwissenschaft. Opladen.

WEINRICH, LOTTE (1992): Verbale und nonverbale Strategien in Fernsehgesprächen. Eine explorative Studie. Tübingen. 